Supporting information for

\title{
One-Pot Transformation of Trichloroacetamide into Readily Deprotectable Carbamates
}

\author{
Toshio Nishikawa, ${ }^{\mathrm{a}, \mathrm{b} *}$ Daisuke Urabe, ${ }^{\mathrm{a}}$ Miho Tomita, ${ }^{\mathrm{a}}$ Takashi Tsujimoto, ${ }^{\mathrm{b}}$ Tomoko Iwabuchi, ${ }^{\mathrm{a}}$ \\ and Minoru Isobe ${ }^{\mathrm{a} *}$
}

${ }^{a}$ Laboratory of Organic Chemistry, School of Bioagricultural Sciences, Nagoya University, Chikusa, Nagoya 464-8601, Japan.

FAX (+)81-52-789-4111; E-mail; nisikawa@agr.nagoya-u.ac.jp

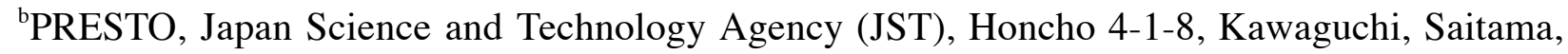
332-0012, Japan

Contents

1. Preparation of substrates

2. Transformation of trichloroacetamide to carbamate 


\section{Preparation of substrates,}

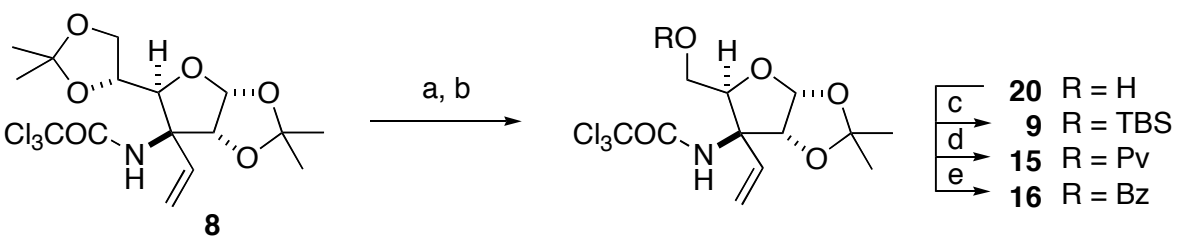

Reagent and Conditions: (a) $\mathrm{H}_{5} \mathrm{IO}_{6}$, AcOEt, rt; (b) $\mathrm{NaBH}_{4}$, EtOH, $0{ }^{\circ} \mathrm{C}$ (90\% in 2 steps); (c) TBSCl, imid. DMF (96\%); (d) PvCl, DMAP, pyridine (97\%); (e) BzCl, pyridine (96\%).

Alcohol 20. $\mathrm{Mp} 95-96^{\circ} \mathrm{C} .[\alpha]_{\mathrm{D}}^{25}+58.7\left(c 1.04, \mathrm{CHCl}_{3}\right)$; IR (film) $v_{\max } 3464,3250,2992,1717$, 1523, 1376, 1217, 1084, $1008 \mathrm{~cm}^{-1}$. ${ }^{1} \mathrm{H} \mathrm{NMR}\left(300 \mathrm{MHz}, \mathrm{CDCl}_{3}\right) \delta 1.36$ (3H, s, acetonide), 1.55 (3H, s, acetonide), $3.94(1 \mathrm{H}, \mathrm{d}, J=3.5 \mathrm{~Hz}, \mathrm{H}-4), 3.96(1 \mathrm{H}, \mathrm{dd}, J=13 \mathrm{~Hz}, \mathrm{H}-5), 4.18(1 \mathrm{H}, \mathrm{dd}, J=$ $13,3.5 \mathrm{~Hz}, \mathrm{H}-5$ ') $5.06(1 \mathrm{H}, \mathrm{d}, J=3.5 \mathrm{~Hz}, \mathrm{H}-2), 5.37\left(1 \mathrm{H}, \mathrm{d}, J=18 \mathrm{~Hz},-\mathrm{CH}=\mathrm{CH}_{\mathrm{A}} \mathrm{H}_{\mathrm{B}}\right), 5.42(1 \mathrm{H}$, $\left.\mathrm{d}, J=11 \mathrm{~Hz},-\mathrm{CH}=\mathrm{CH}_{\mathrm{A}} H_{\mathrm{B}}\right), 5.91(1 \mathrm{H}, \mathrm{d}, J=3.5 \mathrm{~Hz}, \mathrm{H}-1), 6.08(1 \mathrm{H}, \mathrm{dd}, J=18,11 \mathrm{~Hz},-$ $\left.\mathrm{CH}=\mathrm{CH}_{2}\right), 9.57(1 \mathrm{H}$, br s, $-\mathrm{NH}) .{ }^{13} \mathrm{C} \mathrm{NMR}\left(75 \mathrm{MHz}, \mathrm{CDCl}_{3}\right) \delta 26.2,26.7,58.1,70.0,78.3,82.5$, 92.8, 104.5, 112.9, 117.4, 130.0, 161.8. HRMS $\left(\mathrm{FAB}^{+}\right)$calcd. for $\mathrm{C}_{12} \mathrm{H}_{17} \mathrm{O}_{5} \mathrm{NCl}_{3}[\mathrm{M}+\mathrm{H}]$ 360.0173, found. 360.0187 .

TBS-ether 9. $[\alpha]_{\mathrm{D}}^{25}+53\left(c 0.93, \mathrm{CHCl}_{3}\right)$. IR $(\mathrm{KBr}) v_{\max } 3289,2932,2886,2859,1721,1514$, $1473,1375,1255,1217,1166,1086,1013,925,830 \mathrm{~cm}^{-1} .{ }^{1} \mathrm{H}$ NMR $\left(400 \mathrm{MHz}, \mathrm{CDCl}_{3}\right) \delta 0.15$ (3H, s, -SiMe), $0.17(3 \mathrm{H}, \mathrm{s},-\mathrm{SiMe}), 0.92(9 \mathrm{H}, \mathrm{s}, t-\mathrm{Bu}), 1.35(3 \mathrm{H}, \mathrm{s}$, acetonide $), 1.54(3 \mathrm{H}, \mathrm{s}$, acetonide), $3.85(1 \mathrm{H}, \mathrm{d}, J=3 \mathrm{~Hz}, \mathrm{H}-4), 4.02(1 \mathrm{H}, \mathrm{d}, J=12.5 \mathrm{~Hz}, \mathrm{H}-5), 4.15(1 \mathrm{H}, \mathrm{dd}, J=12.5,3$ $\mathrm{Hz}, \mathrm{H}-5$ '), $4.99(1 \mathrm{H}, \mathrm{d}, J=3.5 \mathrm{~Hz}, \mathrm{H}-2), 5.35\left(1 \mathrm{H}, \mathrm{d}, J=17.5 \mathrm{~Hz},-\mathrm{CH}=\mathrm{CH}_{\mathrm{A}} \mathrm{H}_{\mathrm{B}}\right), 5.39(1 \mathrm{H}, \mathrm{d}, J$ $\left.=11 \mathrm{~Hz},-\mathrm{CH}=\mathrm{CH}_{\mathrm{A}} H_{\mathrm{B}}\right), 5.88(1 \mathrm{H}, \mathrm{d}, J=3.5 \mathrm{~Hz}, \mathrm{H}-1), 6.08(1 \mathrm{H}, \mathrm{dd}, J=17.5,11 \mathrm{~Hz},-$ $\left.\mathrm{C} H=\mathrm{CH}_{\mathrm{A}} \mathrm{H}_{\mathrm{B}}\right), 9.18(1 \mathrm{H}$, br s, $-\mathrm{NH}) .{ }^{13} \mathrm{C} \mathrm{NMR}\left(100 \mathrm{MHz}, \mathrm{CDCl}_{3}\right) \delta-5.4,-4.7,19.0,26.1,26.2$, 26.7, 59.7, 70.0, 78.3, 82.6, 93.0, 104.4, 112.4, 116.8, 130.6, 161.4. HRMS $\left(\mathrm{FAB}^{+}\right)$calcd. for $\mathrm{C}_{18} \mathrm{H}_{31} \mathrm{NO}_{5} \mathrm{SiCl}_{3}[\mathrm{M}+\mathrm{H}]$ 474.1038, found 474.1045.

Pivalate 15. $[\alpha]_{\mathrm{D}}^{25}+45\left(c 0.66, \mathrm{CHCl}_{3}\right)$. IR $(\mathrm{KBr}) v_{\max } 3392,2982,1729,1501,1376,1218$, 1084, $1007 \mathrm{~cm}^{-1}$. ${ }^{1} \mathrm{H}$ NMR (400 MHz, $\left.\mathrm{CDCl}_{3}\right) \delta 1.22(9 \mathrm{H}, \mathrm{s}, t-\mathrm{Bu}), 1.35$ (3H, s, acetonide), 1.57 (3H, s, acetonide), $4.12(1 \mathrm{H}, \mathrm{dd}, J=12.5,4 \mathrm{~Hz}, \mathrm{H}-5), 4.27(1 \mathrm{H}, \mathrm{dd}, J=5.5,4.5 \mathrm{~Hz}, \mathrm{H}-4), 4.63$ $(1 \mathrm{H}, \mathrm{dd}, J=12.5,5.5 \mathrm{~Hz}, \mathrm{H}-5$ ') $5.08(1 \mathrm{H}, \mathrm{d}, J=3.5 \mathrm{~Hz}, \mathrm{H}-2), 5.34(1 \mathrm{H}, \mathrm{d}, J=17.5 \mathrm{~Hz},-$ $\left.\mathrm{CH}=\mathrm{CH}_{\mathrm{A}} \mathrm{H}_{\mathrm{B}}\right), 5.40\left(1 \mathrm{H}, \mathrm{d}, J=11 \mathrm{~Hz},-\mathrm{CH}=\mathrm{CH}_{\mathrm{A}} H_{\mathrm{B}}\right), 5.92(1 \mathrm{H}, \mathrm{d}, J=3.5 \mathrm{~Hz}, \mathrm{H}-1), 6.06(1 \mathrm{H}, \mathrm{dd}$, $\left.J=17.5,11 \mathrm{~Hz},-\mathrm{CH}=\mathrm{CH}_{2}\right), 7.30(1 \mathrm{H}$, br s, $-\mathrm{N} H) .{ }^{13} \mathrm{C} \mathrm{NMR}\left(100 \mathrm{MHz}, \mathrm{CDCl}_{3}\right) \delta 26.2,26.6$, 27.2, 38.9, 59.3, 69.2, 78.4, 83.4, 92.9, 104.7, 112.8, 117.4, 130.5, 161.3, 178.1. HRMS (FAB $\left.{ }^{+}\right)$ calcd. for $\mathrm{C}_{17} \mathrm{H}_{25} \mathrm{O}_{6} \mathrm{NCl}_{3}[\mathrm{M}+\mathrm{H}]$ 444.0748, found. 444.0746.

Benzoate 16. $[\alpha]_{\mathrm{D}}^{25}+49.1\left(c 1.59, \mathrm{CHCl}_{3}\right)$. IR $(\mathrm{KBr}) v_{\max } 3391,2990,1728,1603,1508,1376$, 1270, 1165, 1084, 1025, $822 \mathrm{~cm}^{-1}$. ${ }^{1} \mathrm{H}$ NMR (300 MHz, $\left.\mathrm{CDCl}_{3}\right) \delta 1.37$ (3H, s, acetonide), 1.59 (3H, s, acetonide), $4.33(1 \mathrm{H}, \mathrm{dd}, J=4.5,3 \mathrm{~Hz}, \mathrm{H}-4), 4.56(1 \mathrm{H}, \mathrm{dd}, J=13,3 \mathrm{~Hz}, \mathrm{H}-5), 4.80(1 \mathrm{H}$, $\mathrm{dd}, J=13,4.5 \mathrm{~Hz}, \mathrm{H}-5$ '), $5.05(1 \mathrm{H}, \mathrm{d}, J=3.5 \mathrm{~Hz}, \mathrm{H}-2), 5.35\left(1 \mathrm{H}, \mathrm{d}, J=17.5 \mathrm{~Hz},-\mathrm{CH}=\mathrm{CH}_{\mathrm{A}} \mathrm{H}_{\mathrm{B}}\right)$, $5.42\left(1 \mathrm{H}, \mathrm{d}, J=11 \mathrm{~Hz},-\mathrm{CH}=\mathrm{CH}_{\mathrm{A}} H_{\mathrm{B}}\right), 5.97(1 \mathrm{H}, \mathrm{d}, J=3.5 \mathrm{~Hz}, \mathrm{H}-1), 6.10(1 \mathrm{H}, \mathrm{dd}, J=17.5,11$ $\left.\mathrm{Hz},-\mathrm{CH}=\mathrm{CH}_{2}\right), 7.43-7.51(2 \mathrm{H}, \mathrm{m}, \mathrm{Ar}), 7.26(1 \mathrm{H}, \mathrm{tt}, J=7.5,1.5 \mathrm{~Hz}-\mathrm{Ar}), 7.73(1 \mathrm{H}, \mathrm{br} \mathrm{s},-\mathrm{NH})$, 
$8.04(2 \mathrm{H}, \mathrm{m},-\mathrm{Ar}) .{ }^{13} \mathrm{C} \mathrm{NMR}\left(100 \mathrm{MHz}, \mathrm{CDCl}_{3}\right) \delta 26.2,26.6,59.8,69.4,77.8,82.9,92.6,104.5$, 112.8, 117.7, 128.5, 129.1, 129.8, 129.9, 133.6, 161.2, 166.1. HRMS (FAB+) calcd. for $\mathrm{C}_{19} \mathrm{H}_{21} \mathrm{O}_{6} \mathrm{NCl}_{3}[\mathrm{M}+\mathrm{H}]$ 464.0435, found. 464.0432.

\section{Transformation of trichloroacetamide to carbamate}

Dry DMF was purchased as dehydrated DMF from Kanto Chemical Co., Inc. CuCl was dried under vacuum after washing with $\mathrm{EtOH}$ and $\mathrm{Et}_{2} \mathrm{O}{ }^{1}$

\section{Experimental procedure for equation 1.}

Trichloroacetamide 1 (50 mg, $0.13 \mathrm{mmol}$ ) and $\mathrm{Na}_{2} \mathrm{CO}_{3}(29 \mathrm{mg}, 0.26 \mathrm{mmol}$ ) were placed in a reaction vessel, which was filled with nitrogen. DMF $(2.0 \mathrm{ml})$ and benzyl alcohol $(70 \mu 1,0.66$ mmol) were added, and the mixture was stirred at reflux temperature for $2 \mathrm{~h}$. After cooling to rt, the mixture was diluted with $\mathrm{Et}_{2} \mathrm{O}(20 \mathrm{ml})$, and washed with $\mathrm{H}_{2} \mathrm{O}(\mathrm{x} 1)$ and brine $(\mathrm{x} 1)$, dried over anhydrous $\mathrm{Na}_{2} \mathrm{SO}_{4}$, and concentrated. The residue was purified by flash chromatography to give benzyl carbamate $\mathbf{2 a}(47 \mathrm{mg}, 96 \%)$.

\section{Typical experimental procedure exemplified for 1 to 2 a (entry 1 in Table 1).}

A mixture of trichloroacetamide $1(50 \mathrm{mg}, 0.13 \mathrm{mmol})$ and $\mathrm{Na}_{2} \mathrm{CO}_{3}(29 \mathrm{mg}, 0.26 \mathrm{mmol})$ in dry DMF $(2.0 \mathrm{ml}$ ) was heated at reflux temperature for $40 \mathrm{~min}$. (Completion of the reaction was monitored by silica gel TLC). After cooling to rt, $n-\mathrm{Bu}_{4} \mathrm{NCl}$ (75 $\mathrm{mg}, 0.27 \mathrm{mmol}$ ), benzyl alcohol (140 $\mu \mathrm{l}, 1.32 \mathrm{mmol})$ and $\mathrm{CuCl}(27 \mathrm{mg}, 0.27 \mathrm{mmol})$ were added and the resulting mixture was stirred at $\mathrm{rt}$ for $45 \mathrm{~min}$. and then filtered through the pad of Super-Cel. The filtrate was diluted with $\mathrm{Et}_{2} \mathrm{O}$, and the solution was washed with $\mathrm{H}_{2} \mathrm{O}$, sat. $\mathrm{NH}_{4} \mathrm{Cl}$ solution and brine, dried over anhydrous $\mathrm{Na}_{2} \mathrm{SO}_{4}$, and concentrated under reduced pressure. The residue was purified by silica gel column chromatography to give benzyl carbamate $\mathbf{2 a}(41 \mathrm{mg}, 83 \%)$.

2a $(\mathrm{R}=\mathrm{Bn}):[\alpha]_{\mathrm{D}}{ }^{27}+55.1\left(c 1.02, \mathrm{CHCl}_{3}\right)$. IR $(\mathrm{KBr}) v_{\max } 3379,2985,2908,1733,1518,1255$, $1063 \mathrm{~cm}^{-1}$. ${ }^{1} \mathrm{H}$ NMR $\left(\mathrm{CDCl}_{3}, 300 \mathrm{MHz}\right) \delta 1.38\left(3 \mathrm{H}, \mathrm{s}, \mathrm{CH}_{3}\right.$ of acetonide $), 1.42\left(3 \mathrm{H}, \mathrm{s}, \mathrm{CH}_{3}\right.$ of acetonide), 1.61-1.69 $(5 \mathrm{H}, \mathrm{m}), 2.01(1 \mathrm{H}, \mathrm{m}), 2.28\left(1 \mathrm{H}\right.$, br d, $\left.J=18 \mathrm{~Hz}, \mathrm{CH}_{\mathrm{A}} \mathrm{H}_{\mathrm{B}}\right), 3.22(1 \mathrm{H}$, br d, $\left.J=18 \mathrm{~Hz}, \mathrm{CH}_{\mathrm{A}} H_{B}\right), 3.60(1 \mathrm{H}, \mathrm{m}), 3.94-4.06(2 \mathrm{H}, \mathrm{m}), 5.07\left(2 \mathrm{H}\right.$, br s, $\left.-\mathrm{CH}_{2}-\mathrm{Ph}\right), 5.22(1 \mathrm{H}, \mathrm{dd}, J=$ $\left.17.5,1 \mathrm{~Hz},-\mathrm{CH}=\mathrm{CH}_{\mathrm{A}} \mathrm{H}_{\mathrm{B}}\right), 5.26\left(1 \mathrm{H}\right.$, br d, $\left.J=11 \mathrm{~Hz},-\mathrm{CH}=\mathrm{CH}_{\mathrm{A}} H_{\mathrm{B}}\right), 5.37\left(1 \mathrm{H}, \mathrm{br},-\mathrm{CH}=\mathrm{C}-\mathrm{CH}_{3}\right)$, $5.85\left(1 \mathrm{H}, \mathrm{dd}, J=17.5,11 \mathrm{~Hz},-\mathrm{CH}=\mathrm{CH}_{2}\right), 7.18(1 \mathrm{H}$, br s, $-\mathrm{NH}), 7.27-7.38(5 \mathrm{H}, \mathrm{m}, P h) .{ }^{13} \mathrm{C}$ $\mathrm{NMR}\left(\mathrm{CDCl}_{3}, 75 \mathrm{MHz}\right) \delta 22.6,26.1,26.5,30.2,36.8,44.2,58.0,65.6,68.7,109.7,115.3,119.5$, 127.7, 128.4, 130.8, 136.2, 137.4, 155.0. EI-MS $m / z 371\left(\mathrm{M}^{+}\right), 356(\mathrm{M}-15)$. Anal. Calcd for $\mathrm{C}_{22} \mathrm{H}_{29} \mathrm{O}_{4} \mathrm{~N}_{1}: \mathrm{C}, 71.13 ; \mathrm{H}, 7.87 ; \mathrm{N}, 3.77$. Found: C, 70.93; H, 7.97; N, 3.81.

2b $\left(\mathrm{R}=\mathrm{CH}_{2} \mathrm{CCl}_{3}\right):[\alpha]_{\mathrm{D}}{ }^{28}+43.5\left(c 1.75, \mathrm{CHCl}_{3}\right) . \quad \mathrm{IR}(\mathrm{KBr}) v_{\max } 3363,2986,2908,1752,1523$, $1256,1238,1109,1066 \mathrm{~cm}^{-1}$. ${ }^{1} \mathrm{H} \mathrm{NMR}\left(\mathrm{CDCl}_{3}, 300 \mathrm{MHz}\right) \delta 1.38$ (3H, s, $\mathrm{CH}_{3}$ of acetonide), 1.44 (3H, s, $\mathrm{CH}_{3}$ of acetonide), 1.62-1.72 $(5 \mathrm{H}, \mathrm{m}), 2.02(1 \mathrm{H}, \mathrm{m}), 2.28\left(1 \mathrm{H}, \mathrm{br} \mathrm{d}, J=18 \mathrm{~Hz}, \mathrm{CH}_{A} \mathrm{H}_{\mathrm{B}}\right)$, $3.18\left(1 \mathrm{H}\right.$, br dd, $\left.J=18,6 \mathrm{~Hz}, \mathrm{CH}_{\mathrm{A}} H_{\mathrm{B}}\right), 3.62(1 \mathrm{H}, \mathrm{t}, J=8 \mathrm{~Hz}), 3.94-4.09(2 \mathrm{H}, \mathrm{m}), 4.57(1 \mathrm{H}, \mathrm{d}, J=$

\footnotetext{
${ }^{1}$ Perrin, D. D.; Armarego, W. L. F. Purification of Laboratory Chemicals, 3rd ed., Pergamon Press, 1988, pp 322.
} 
$\left.12 \mathrm{~Hz},-\mathrm{CH}_{\mathrm{A}} \mathrm{H}_{\mathrm{B}} \mathrm{CCl}_{3}\right), 4.77\left(1 \mathrm{H}, \mathrm{d}, J=12 \mathrm{~Hz},-\mathrm{CH}_{\mathrm{A}} H_{\mathrm{B}} \mathrm{CCl}_{3}\right), 5.28(1 \mathrm{H}, \mathrm{dd}, J=18,1 \mathrm{~Hz},-$ $\left.\mathrm{CH}=\mathrm{CH}_{A} \mathrm{H}_{\mathrm{B}}\right), 5.29\left(1 \mathrm{H}\right.$, br d, $\left.J=10 \mathrm{~Hz},-\mathrm{CH}=\mathrm{CH}_{\mathrm{A}} H_{\mathrm{B}}\right), 5.38\left(1 \mathrm{H}, \mathrm{br},-\mathrm{CH}=\mathrm{C}-\mathrm{CH}_{3}\right), 5.84(1 \mathrm{H}, \mathrm{dd}$, $\left.J=18,10 \mathrm{~Hz},-\mathrm{CH}=\mathrm{CH}_{2}\right), 7.59(1 \mathrm{H}$, br s, $-\mathrm{NH}) .{ }^{13} \mathrm{C} \mathrm{NMR}\left(\mathrm{CDCl}_{3}, 75 \mathrm{MHz}\right) \delta 22.7,26.0,26.5$, 30.2, 36.6, 44.2, 58.2, 68.8, 73.8, 76.7, 109.7, 115.6, 119.2, 130.9, 135.5, 152.9. EI-MS $\mathrm{m} / z .415$ $\left(\mathrm{M}^{+}\right), 413\left(\mathrm{M}^{+}\right), 411\left(\mathrm{M}^{+}\right), 400(\mathrm{M}-15), 398(\mathrm{M}-15), 396(\mathrm{M}-15)$. HRMS (EI) Calcd for $\mathrm{C}_{17} \mathrm{H}_{24} \mathrm{NO}_{4} \mathrm{Cl}_{3}\left(\mathrm{M}^{+}\right)$411.0771. Found 411.0736.

2c $\left(\mathrm{R}=\mathrm{CH}_{2} \mathrm{CH}=\mathrm{CH}_{2}\right):[\alpha]_{\mathrm{D}}{ }^{27}+60\left(c 0.81, \mathrm{CHCl}_{3}\right)$. IR $(\mathrm{KBr}) v_{\max } 3384,2986,2907,1733,1521$, $1255,1066 \mathrm{~cm}^{-1} .{ }^{1} \mathrm{H}$ NMR $\left(\mathrm{CDCl}_{3}, 300 \mathrm{MHz}\right) \delta 1.39\left(3 \mathrm{H}, \mathrm{s}, \mathrm{CH}_{3}\right.$ of acetonide $), 1.43\left(3 \mathrm{H}, \mathrm{s}, \mathrm{CH}_{3}\right.$ of acetonide), 1.61-1.72 $(5 \mathrm{H}, \mathrm{m}), 2.00(1 \mathrm{H}, \mathrm{m}), 2.27\left(1 \mathrm{H}, \mathrm{br} \mathrm{d}, J=18 \mathrm{~Hz}, \mathrm{CH}_{A} \mathrm{H}_{\mathrm{B}}\right), 3.22(1 \mathrm{H}, \mathrm{br}$ d, $\left.J=18 \mathrm{~Hz}, \mathrm{CH}_{\mathrm{A}} H_{\mathrm{B}}\right), 3.60(1 \mathrm{H}, \mathrm{m}), 3.94-4.07(2 \mathrm{H}, \mathrm{m}), 4.52\left(2 \mathrm{H}, \mathrm{br} \mathrm{d}, J=5 \mathrm{~Hz},-\mathrm{CH}_{2^{-}}\right.$ $\left.\mathrm{CH}=\mathrm{CH}_{2}\right), 5.15-5.34\left(4 \mathrm{H}, \mathrm{m},-\mathrm{CH}=\mathrm{CH}_{2} \times 2\right), 5.37\left(1 \mathrm{H}\right.$, br d, $\left.\mathrm{J}=4 \mathrm{~Hz},-\mathrm{CH}=\mathrm{C}-\mathrm{CH}_{3}\right), 5.85(1 \mathrm{H}$, $\left.\mathrm{dd}, J=17,11 \mathrm{~Hz},-\mathrm{C}-\mathrm{C} H=\mathrm{CH}_{2}\right), 5.77-6.01\left(1 \mathrm{H}, \mathrm{m},-\mathrm{CH}_{2}-\mathrm{CH}=\mathrm{CH}_{2}\right), 7.15(1 \mathrm{H}$, br s, $-\mathrm{NH}) .{ }^{13} \mathrm{C}$ $\operatorname{NMR}\left(\mathrm{CDCl}_{3}, 75 \mathrm{MHz}\right) \delta 22.6,26.1,26.5,30.2,36.8,44.2,57.9,64.6,68.7,109.7,115.3,116.8$, 119.5, 130.7, 133.6, 136.2, 154.9. EI-MS m/z $321\left(\mathrm{M}^{+}\right), 306(\mathrm{M}-15)$. HRMS (EI) Calcd for $\mathrm{C}_{18} \mathrm{H}_{27} \mathrm{NO}_{4}\left(\mathrm{M}^{+}\right)$321.1940. Found 321.1927.

2d $(\mathrm{R}=t-\mathrm{Bu}):[\alpha]_{\mathrm{D}}^{29}+21.1\left(c 2.46, \mathrm{CH}_{2} \mathrm{Cl}_{2}\right)$. IR ( $\left.\mathrm{KBr}\right) v_{\max } 3394,2980,2902,1727,1515,1367$, 1254, 1174, 1066, $862 \mathrm{~cm}^{-1}$. ${ }^{1} \mathrm{H}$ NMR $\left(\mathrm{CDCl}_{3}, 300 \mathrm{MHz}\right) \delta 1.38\left(3 \mathrm{H}, \mathrm{s}, \mathrm{CH}_{3}\right), 1.43(9 \mathrm{H}, \mathrm{s}, t-\mathrm{Bu})$, 1.60-1.71 (8H, m, $\mathrm{CH}_{3}$ x 2, C-CH $\left.-\mathrm{CH}\right), 1.92-2.06(1 \mathrm{H}, \mathrm{m}, \mathrm{CH}-\mathrm{CH}-\mathrm{O}), 2.27(1 \mathrm{H}, \mathrm{br} \mathrm{d}, J=17$ $\left.\mathrm{Hz}, \mathrm{C}=\mathrm{CH}-\mathrm{CH}_{A} \mathrm{H}_{\mathrm{B}}\right), 3.18\left(1 \mathrm{H}\right.$, br d, $\left.J=17 \mathrm{~Hz}, \mathrm{CH}=\mathrm{CH}-\mathrm{CH}_{\mathrm{A}} H_{B}\right), 3.59(1 \mathrm{H}, \mathrm{td}, J=7,1.5 \mathrm{~Hz},-\mathrm{O}-$ $\left.\mathrm{CH}_{A} \mathrm{H}_{\mathrm{B}}-\mathrm{CH}-\mathrm{O}-\right), 3.93-4.06\left(2 \mathrm{H}, \mathrm{m},-\mathrm{O}-\mathrm{CH}_{\mathrm{A}} H_{B}-\mathrm{CH}-\mathrm{O}-\right), 5.24\left(1 \mathrm{H}, \mathrm{dd}, J=17,1.5 \mathrm{~Hz}, \mathrm{CH}=H_{A} \mathrm{H}_{\mathrm{B}}\right)$, $5.26\left(1 \mathrm{H}, \mathrm{br} \mathrm{d}, J=10.5 \mathrm{~Hz}, \mathrm{CH}=\mathrm{CH}_{\mathrm{A}} H_{B}\right), 5.33-5.40(1 \mathrm{H}, \mathrm{m}, \mathrm{CH}=\mathrm{C}), 5.84(1 \mathrm{H}, \mathrm{dd}, J=17,10.5$ $\left.\mathrm{Hz}, \mathrm{CH}=\mathrm{CH}_{2}\right), 6.88(1 \mathrm{H}$, br s, $\mathrm{NH}){ }^{13} \mathrm{C} \mathrm{NMR}\left(\mathrm{CDCl}_{3}, 100 \mathrm{MHz}\right) \delta 22.7,26.2,26.6,28.5,30.3$, 37.1, 44.2, 57.8, 68.7, 78.3, 109.5, 114.9, 119.6, 130.6, 136.6, 154.9. Anal. Calcd for $\mathrm{C}_{19} \mathrm{H}_{31} \mathrm{O}_{4} \mathrm{~N}$ : C, 67.63; H, 9.26; N, 4.15. Found: C, 67.63; H, 9.26; N, 4.35.

2e $\left(\mathrm{R}=\mathrm{CH}_{2} \mathrm{CH}_{2} \mathrm{TMS}\right) . \quad[\alpha]_{\mathrm{D}}^{29}+54.1\left(c 1.38, \mathrm{CHCl}_{3}\right)$. IR $(\mathrm{KBr}) v_{\max } 3386,2956,1733,1525$, $1372,1255,1063,844 \mathrm{~cm}^{-1} .{ }^{1} \mathrm{H}$ NMR $\left(\mathrm{CDCl}_{3}, 300 \mathrm{MHz}\right) \delta 0.04(9 \mathrm{H}, \mathrm{s}, \mathrm{TMS}), 0.96(2 \mathrm{H}, \mathrm{t}, J=8$ $\left.\mathrm{Hz}, \mathrm{Si}-\mathrm{CH}_{2}\right), 1.38\left(3 \mathrm{H}, \mathrm{s}, \mathrm{CH}_{3}\right), 1.42\left(3 \mathrm{H}, \mathrm{s}, \mathrm{CH}_{3}\right), 1.50-1.72\left(2 \mathrm{H}, \mathrm{m}, \mathrm{CH}_{2}\right), 1.63\left(3 \mathrm{H}, \mathrm{s}, \mathrm{CH}_{3}\right)$, 1.91-2.03 $(1 \mathrm{H}, \mathrm{m}, \mathrm{CH}), 2.24\left(1 \mathrm{H}\right.$, br d, $\left.J=18 \mathrm{~Hz}, \mathrm{CH}_{\mathrm{A}} \mathrm{H}_{\mathrm{B}}\right), 3.24\left(1 \mathrm{H}\right.$, br d, $\left.J=18 \mathrm{~Hz}, \mathrm{CH}_{\mathrm{A}} H_{B}\right)$, $3.59\left(1 \mathrm{H}, \mathrm{td}, J=7.5,1 \mathrm{~Hz}, \mathrm{O}-\mathrm{CH}_{A} \mathrm{H}_{\mathrm{B}}-\mathrm{CH}-\mathrm{O}\right), 3.92-4.20\left(4 \mathrm{H}, \mathrm{m}, \mathrm{COO}-\mathrm{CH}_{2}, \mathrm{O}-\mathrm{CH}_{\mathrm{A}} H_{B}-\mathrm{CH}-\mathrm{O}\right)$, $5.24\left(1 \mathrm{H}, \mathrm{dd}, J=17,1.2 \mathrm{~Hz}, \mathrm{CH}=\mathrm{CH}_{A} \mathrm{H}_{\mathrm{B}}\right), 5.27\left(1 \mathrm{H}, \mathrm{dd}, J=10.5,1.5 \mathrm{~Hz}, \mathrm{CH}=\mathrm{CH}_{\mathrm{A}} H_{B}\right), 5.33-$ $5.40(1 \mathrm{H}, \mathrm{m}, \mathrm{C}=\mathrm{CH}), 5.84\left(1 \mathrm{H}, \mathrm{dd}, J=17,10.5 \mathrm{~Hz}, \mathrm{CH}=\mathrm{CH}_{\mathrm{A}} \mathrm{H}_{\mathrm{B}}\right), 7.06(1 \mathrm{H}$, br s, $\mathrm{N} H) .{ }^{13} \mathrm{C}$ NMR $\left(\mathrm{CDCl}_{3}, 75 \mathrm{MHz}\right) \delta-1.4,17.3,22.6,26.2,26.5,30.2,36.9,44.3,57.8,62.0,68.7,109.7$, 115.2, 119.6, 130.7, 136.3, 155.4. Anal. Calcd for $\mathrm{C}_{20} \mathrm{H}_{35} \mathrm{O}_{4} \mathrm{NSi}$ : C, 62.95; H, 9.25; N, 3.67. Found: C, 62.96; H, 9.26; N, 3.58.

2f $\left(\mathrm{R}=\mathrm{CH}_{2} \mathrm{C}_{12} \mathrm{H}_{8}\right) .[\alpha]_{\mathrm{D}}{ }^{28}+50.6\left(c 2.13, \mathrm{CHCl}_{3}\right) . \mathrm{IR}(\mathrm{KBr}) v_{\max } 3379,2898,1736,1526,1450$, $1255 \mathrm{~cm}^{-1} .{ }^{1} \mathrm{H} \mathrm{NMR}\left(\mathrm{CDCl}_{3}, 300 \mathrm{MHz}\right) \delta 1.44\left(3 \mathrm{H}\right.$, br s, $\left.\mathrm{CH}_{3}\right), 1.45\left(3 \mathrm{H}\right.$, br s, $\left.\mathrm{CH}_{3}\right), 1.64(3 \mathrm{H}$, br s, $\left.\mathrm{CH}_{3}\right), 1.54-1.76(2 \mathrm{H}, \mathrm{m}), 2.00(1 \mathrm{H}, \mathrm{m}), 2.28\left(1 \mathrm{H}\right.$, br d, $\left.J=17 \mathrm{~Hz}, \mathrm{CH}_{A} \mathrm{H}_{\mathrm{B}}\right), 3.24(1 \mathrm{H}$, br d, $J$ $\left.=17 \mathrm{~Hz}, \mathrm{CH}_{\mathrm{A}} H_{B}\right), 3.62\left(1 \mathrm{H}\right.$, br t, $\left.J=7.5 \mathrm{~Hz}, \mathrm{O}-\mathrm{CH}_{A} \mathrm{H}_{\mathrm{B}} \mathrm{CH}-\mathrm{O}\right), 3.94-4.10\left(2 \mathrm{H}, \mathrm{m}, \mathrm{O}-\mathrm{CH}_{\mathrm{A}} H_{B^{-}}\right.$ $\mathrm{CH}-\mathrm{O}), 4.14-4.26\left(2 \mathrm{H}, \mathrm{m}, \mathrm{CO}-\mathrm{O}-\mathrm{CH}_{a} \mathrm{H}_{\mathrm{B}}-\mathrm{CH}\right), 4.46\left(1 \mathrm{H}\right.$, br dd, $J=13.0,9 \mathrm{~Hz}, \mathrm{CO}-\mathrm{O}-\mathrm{CH}_{\mathrm{a}} H_{B^{-}}$ $\mathrm{CH}), 5.18\left(1 \mathrm{H}\right.$, br d, $\left.J=17 \mathrm{~Hz}, \mathrm{CH}=\mathrm{CH}_{A} \mathrm{H}_{\mathrm{B}}\right), 5.25\left(1 \mathrm{H}\right.$, br d, $\left.J=10.5 \mathrm{~Hz}, \mathrm{CH}=\mathrm{CH}_{\mathrm{A}} H_{B}\right), 5.38$ $(1 \mathrm{H}, \mathrm{m}, \mathrm{C}=\mathrm{C} H), 5.84\left(1 \mathrm{H}, \mathrm{dd}, J=17,10.5 \mathrm{~Hz}, \mathrm{CH}=\mathrm{CH}_{\mathrm{A}} \mathrm{H}_{\mathrm{B}}\right), 7.30(2 \mathrm{H}, \mathrm{td}, J=7.5,1 \mathrm{~Hz}$, aromatic), 7.34-7.44 (1H, br, $\mathrm{NH}), 7.39(2 \mathrm{H}, \mathrm{td}, J=7.5,0.8 \mathrm{~Hz}$, aromatic $), 7.63(2 \mathrm{H}, \mathrm{br} \mathrm{d}, J=$ $7.5 \mathrm{~Hz}$, aromatic), $7.76\left(2 \mathrm{H}, \mathrm{d}, J=7.5 \mathrm{~Hz}\right.$, aromatic). ${ }^{13} \mathrm{C} \mathrm{NMR}\left(\mathrm{CDCl}_{3}, 100 \mathrm{MHz}\right) \delta 22.7,26.3$, 
26.6, 30.3, 36.9, 44.4, 47.4, 58.0, 65.7, 68.9, 76.8, 109.6, 115.4, 119.4, 119.9, 125.1, 126.9, 127.5, 130.7, 135.9, 141.4, 144.2, 144.3, 154.7. Anal. Calcd for $\mathrm{C}_{29} \mathrm{H}_{33} \mathrm{O}_{4} \mathrm{~N}: \mathrm{C}, 75.79 ; \mathrm{H}, 7.24$; N, 3.05. Found: C, 75.80; H, 7.45; N, 3.01.

Urea 4. $[\alpha]_{\mathrm{D}}{ }^{28}+124\left(c 0.83, \mathrm{CHCl}_{3}\right)$. IR $(\mathrm{KBr}) v_{\max } 3388,2984,2910,1699,1668,1645,1521$, $1371,1067 \mathrm{~cm}^{-1}$. ${ }^{1} \mathrm{H} \mathrm{NMR}\left(\mathrm{CDCl}_{3}, 300 \mathrm{MHz}\right) \delta 1.38\left(3 \mathrm{H} \mathrm{x} \mathrm{2,} \mathrm{s,} \mathrm{CH}_{3}\right.$ of acetonide), $1.40(3 \mathrm{H} \mathrm{x}$ 2, s, $\mathrm{CH}_{3}$ of acetonide), 1.56-1.78 $(5 \mathrm{H} \mathrm{x} \mathrm{2,} \mathrm{m),} 2.01(1 \mathrm{H} \mathrm{x} \mathrm{2,} \mathrm{m),} 2.22(1 \mathrm{H} \mathrm{x} 2, \mathrm{br} \mathrm{d}, J=18 \mathrm{~Hz}$, $\left.\mathrm{CH}_{\mathrm{A}} \mathrm{H}_{\mathrm{B}}\right), 3.30\left(1 \mathrm{H} \mathrm{x} \mathrm{2,} \mathrm{br} \mathrm{dd,} J=18,5.5 \mathrm{~Hz}, \mathrm{CH}_{\mathrm{A}} H_{B}\right), 3.57(1 \mathrm{Hx} 2, \mathrm{~m}), 3.93-4.04(2 \mathrm{Hx} 2, \mathrm{~m}), 5.28$ $\left(1 \mathrm{Hx} 2, \mathrm{dd}, J=10.5,1.5 \mathrm{~Hz},-\mathrm{CH}=\mathrm{CH}_{\mathrm{A}} \mathrm{H}_{\mathrm{B}}\right), 5.30-5.38\left(1 \mathrm{Hx} 2, \mathrm{br},-\mathrm{CH}=\mathrm{C}-\mathrm{CH}_{3}\right), 5.32(1 \mathrm{Hx} 2, \mathrm{dd}, J$ $\left.=17.5,1.5 \mathrm{~Hz},-\mathrm{CH}=\mathrm{CH}_{\mathrm{A}} H_{B}\right), 5.85\left(1 \mathrm{Hx} 2, \mathrm{dd}, J=17.5,10.5 \mathrm{~Hz},-\mathrm{CH}=\mathrm{CH}_{2}\right), 6.38(1 \mathrm{Hx} 2$, br s, $\mathrm{NH}) .{ }^{13} \mathrm{C} \mathrm{NMR}\left(\mathrm{CDCl}_{3}, 75 \mathrm{MHz}\right) \delta 22.6,26.4,26.8,30.3,37.4,44.6,58.0,68.7,76.4,109.5$, 115.5, 119.7, 130.7, 137.2, 156.4. EI-MS m/z $500\left(\mathrm{M}^{+}\right), 485$ (M-15). HRMS (EI) Calcd for $\mathrm{C}_{29} \mathrm{H}_{44} \mathrm{~N}_{2} \mathrm{O}_{5}\left(\mathrm{M}^{+}\right)$500.3250. Found 500.3206.

Compound 10. IR (KBr) $v_{\max } 3363,2972,1729,1507,1238,1073,914 \mathrm{~cm}^{-1} .{ }^{1} \mathrm{H} \mathrm{NMR}\left(\mathrm{CDCl}_{3}\right.$, $300 \mathrm{MHz}) \delta 1.41\left(3 \mathrm{H}, \mathrm{s}, \mathrm{NH}-\mathrm{C}-\mathrm{CH}_{3}\right), 1.57\left(3 \mathrm{H}\right.$, br s, $\left.\mathrm{CH}=\mathrm{C}-\mathrm{CH}_{3}\right), 1.67(3 \mathrm{H}, \mathrm{d}, J=1.0 \mathrm{~Hz}$, $\left.\mathrm{CH}=\mathrm{C}-\mathrm{CH}_{3}\right), 1.67-1.74\left(2 \mathrm{H}, \mathrm{m}, \mathrm{CH}_{2}\right), 1.89-2.00\left(2 \mathrm{H}, \mathrm{m}, \mathrm{CH}_{2}\right), 4.85(1 \mathrm{H}, \mathrm{s}, \mathrm{NH}), 5.04-5.14(1 \mathrm{H}$, $\left.\mathrm{m}, \mathrm{C}=\mathrm{CH}-\mathrm{CH}_{2}\right), 5.06\left(2 \mathrm{H}, \mathrm{s}, \mathrm{CH}_{2}-\mathrm{Ph}\right), 5.09\left(1 \mathrm{H}, \mathrm{d}, J=17.5 \mathrm{~Hz}, \mathrm{CH}=\mathrm{CH}_{A} \mathrm{CH}_{\mathrm{B}}\right), 5.11(1 \mathrm{H}, \mathrm{d}, J=$ $\left.10.5 \mathrm{~Hz}, \mathrm{CH}=\mathrm{CH}_{\mathrm{A}} \mathrm{CH}_{B}\right), 5.90\left(1 \mathrm{H}, \mathrm{dd}, J=17.5,10.5 \mathrm{~Hz}, \mathrm{CH}=\mathrm{CH}_{\mathrm{A}} \mathrm{CH}_{\mathrm{B}}\right), 7.27-7.40(5 \mathrm{H}, \mathrm{m}, \mathrm{Ph})$. ${ }^{13} \mathrm{C} \mathrm{NMR}\left(\mathrm{CDCl}_{3}, 100 \mathrm{MHz}\right) \delta 17.6,22.5,24.5,25.6,39.6,56.5,66.1,112.4,123.8,128.0$, 128.1, 128.5, 131.9, 136.7, 143.1, 154.5. FAB-MS $m / z 288(\mathrm{M}+\mathrm{H})^{+}$. Anal. Calcd for $\mathrm{C}_{18} \mathrm{H}_{25} \mathrm{O}_{2} \mathrm{~N}$ : C, 75.22; H, 8.77; N, 4.87. Found: C, 75.23; H, 8.67; N, 5.08.

Compound 11. $[\alpha]_{27}^{\mathrm{D}}-33.8\left(c 1.82, \mathrm{CHCl}_{3}\right)$. IR (KBr) $v_{\text {max }} 3343,2956,2928,2857,1726$, $1510,1308 \mathrm{~cm}^{-1}$. ${ }^{1} \mathrm{H}$ NMR $\left(\mathrm{CDCl}_{3}, 300 \mathrm{MHz}\right) \delta 0.06\left(6 \mathrm{H}, \mathrm{s},-\mathrm{OSi}\left(\mathrm{CH}_{3}\right)_{2}\right), 0.89\left(9 \mathrm{H}, \mathrm{s}, \mathrm{C}\left(\mathrm{CH}_{3}\right)_{3}\right)$, $1.21\left(3 \mathrm{H}, \mathrm{t}, J=7 \mathrm{~Hz},-\mathrm{OCH}_{2} \mathrm{CH}_{3}\right), 3.49-3.65(2 \mathrm{H}, \mathrm{m}), 3.71(1 \mathrm{H}, \mathrm{dd}, J=10,6 \mathrm{~Hz}), 3.81(1 \mathrm{H}, \mathrm{dq}$, $\left.J=10,7 \mathrm{~Hz}, \mathrm{CH}_{A} \mathrm{H}_{\mathrm{B}}\right), 4.12(1 \mathrm{H}, \mathrm{m}), 4.45(1 \mathrm{H}, \mathrm{m}), 4.94(1 \mathrm{H}, \mathrm{d}, J=4 \mathrm{~Hz}), 5.10-5.17(1 \mathrm{H}, \mathrm{br})$, $5.12\left(2 \mathrm{H}\right.$, br s, $\left.-\mathrm{CH}_{2}-\mathrm{Ph}\right), 5.62\left(1 \mathrm{H}\right.$, br d, $\left.J=10.5 \mathrm{~Hz}, \mathrm{CH}_{\mathrm{A}}=\mathrm{CH}_{\mathrm{B}}\right), 5.84(1 \mathrm{H}$, br d, $J=10.5 \mathrm{~Hz}$, $\left.\mathrm{CH}_{\mathrm{A}}=\mathrm{CH}_{\mathrm{B}}\right), 7.28-7.40(5 \mathrm{H}, \mathrm{m}, \mathrm{Ph}) .{ }^{13} \mathrm{C} \mathrm{NMR}\left(\mathrm{CDCl}_{3}, 75 \mathrm{MHz}\right) \delta-5.5,-5.4,14.9,18.2,25.8$, 47.3, 63.7, 65.4, 66.8, 68.8, 95.6, 125.3, 128.0, 128.2, 128.6, 136.5, 156.1. Anal. Calcd for $\mathrm{C}_{22} \mathrm{H}_{35} \mathrm{O}_{5} \mathrm{NSi}$ : C, 62.68; H, 8.37; N, 3.32. Found: C, 62.68; H, 8.50; N, 3.41.

Compound 12. $[\alpha]^{\mathrm{D}}{ }_{27}+23.9\left(c 1.50, \mathrm{CHCl}_{3}\right) . \quad \mathrm{IR}(\mathrm{KBr}) v_{\max } 3330,2922,1717,1695,1522$, $1251 \mathrm{~cm}^{-1}$. ${ }^{1} \mathrm{H}$ NMR $\left(\mathrm{CDCl}_{3}, 300 \mathrm{MHz}\right) \delta 0.75\left(3 \mathrm{H}, \mathrm{s}, \mathrm{CH}_{3}\right), 1.17(1 \mathrm{H}, \mathrm{d}, J=10 \mathrm{~Hz}), 1.26(3 \mathrm{H}$, s, $\left.\mathrm{CH}_{3}\right), 1.79(1 \mathrm{H}$, br dt, $J=14.5,3 \mathrm{~Hz}), 2.01(1 \mathrm{H}, \mathrm{m}), 2.40-2.55(3 \mathrm{H}, \mathrm{m}), 4.53(1 \mathrm{H}, \mathrm{br}), 4.83$ $\left(1 \mathrm{H}\right.$, br s, C=CH $\left.H_{\mathrm{A}} \mathrm{H}_{\mathrm{B}}\right), 4.93(1 \mathrm{H}, \mathrm{br}, \mathrm{NH}), 5.02\left(1 \mathrm{H}, \mathrm{t}, J=1.5 \mathrm{~Hz}, \mathrm{C}=\mathrm{CH}_{\mathrm{A}} H_{\mathrm{B}}\right), 5.11(2 \mathrm{H}$, br s, $\left.\mathrm{CH}_{2} \mathrm{-Ph}\right), 7.27-7.42(5 \mathrm{H}, \mathrm{m}, \mathrm{Ph}) .{ }^{13} \mathrm{C} \mathrm{NMR}\left(\mathrm{CDCl}_{3}, 75 \mathrm{MHz}\right) \delta 22.0,25.8,29.7,34.8,39.8,40.3$, 46.2, 51.3, 66.6, 111.7, 128.1, 128.6, 136.6, 153.4, 156.0. EI-MS m/z $285\left(\mathrm{M}^{+}\right), 194$ (M-91). Anal. Calcd for $\mathrm{C}_{18} \mathrm{H}_{23} \mathrm{O}_{2} \mathrm{Si}$ : C, 75.76; H, 8.12; N, 4.91. Found: C, 75.67; H, 8.32; N, 5.03.

Benzyl Carbamate 13. $[\alpha]_{\mathrm{D}}^{27}+41\left(c 0.54, \mathrm{CHCl}_{3}\right)$. IR $(\mathrm{KBr}) v_{\max } 3358,2989,2938,2897$, 1736, 1509, 1374, 1216, 1166, 1074, $875 \mathrm{~cm}^{-1}$. ${ }^{1} \mathrm{H}$ NMR (400 MHz, $\left.\mathrm{CDCl}_{3}\right) \delta 1.33(6 \mathrm{H}, \mathrm{s}$, acetonide), $1.43(3 \mathrm{H}, \mathrm{s}$, acetonide), $1.54(3 \mathrm{H}, \mathrm{s}$, acetonide), $4.02(2 \mathrm{H}, \mathrm{d}, J=7 \mathrm{~Hz}, \mathrm{H}-6), 4.08$ $(1 \mathrm{H}, \mathrm{d}, J=5 \mathrm{~Hz}, \mathrm{H}-4), 4.39(1 \mathrm{H}, \mathrm{td}, J=7,5 \mathrm{~Hz}, \mathrm{H}-5), 5.05\left(1 \mathrm{H}, \mathrm{d}, J=12.5 \mathrm{~Hz},-\mathrm{CH}_{\mathrm{A}} \mathrm{H}_{\mathrm{B}} \mathrm{Ph}\right)$, $5.13\left(1 \mathrm{H}, \mathrm{d}, J=12.5 \mathrm{~Hz},-\mathrm{CH}_{\mathrm{A}} H_{\mathrm{B}} \mathrm{Ph}\right), 5.23(1 \mathrm{H}$, br s, H-2), $5.34(1 \mathrm{H}, \mathrm{d}, J=11 \mathrm{~Hz},-$ $\left.\mathrm{CH}=\mathrm{CH}_{\mathrm{A}} \mathrm{H}_{\mathrm{B}}\right), 5.34\left(1 \mathrm{H}, \mathrm{d}, J=17 \mathrm{~Hz},-\mathrm{CH}=\mathrm{CH}_{\mathrm{A}} H_{\mathrm{B}}\right), 5.92(1 \mathrm{H}, \mathrm{d}, J=3.5 \mathrm{~Hz}, \mathrm{H}-1), 6.04(1 \mathrm{H}, \mathrm{dd}$, $\left.J=17,11 \mathrm{~Hz},-\mathrm{CH}=\mathrm{CH}_{2}\right), 6.33(1 \mathrm{H}$, br s, $-\mathrm{NH}), 7.29-7.39(5 \mathrm{H}, \mathrm{m},-\mathrm{Ar}) .{ }^{13} \mathrm{C} \mathrm{NMR}(100 \mathrm{MHz}$, $\left.\mathrm{CDCl}_{3}\right) \delta 24.8,26.2,26.2,26.8,66.0,66.6,68.3,74.7,80.0,84.8,104.4,110.1,112.1,116.9$, 
127.9, 128.1, 128.5, 133.2, 136.4, 155.2. HRMS $\left(\mathrm{FAB}^{+}\right)$calcd. for $\mathrm{C}_{22} \mathrm{H}_{30} \mathrm{NO}_{7}[\mathrm{M}+\mathrm{H}]$ 420.2022, found 420.2024 .

Benzyl Carbamate 14. $[\alpha]_{\mathrm{D}}^{27}+56\left(c 0.98, \mathrm{CHCl}_{3}\right)$. IR $(\mathrm{KBr}) v_{\max } 3354,2931,1732,1509$, 1374, 1255, 1216, 1059, 1016, $836 \mathrm{~cm}^{-1} .{ }^{1} \mathrm{H}$ NMR (400 MHz, $\left.\mathrm{CDCl}_{3}\right) \delta 0.08$ (3H, s, -SiMe), $0.10(3 \mathrm{H}, \mathrm{s},-\mathrm{SiMe}), 0.87(9 \mathrm{H}, \mathrm{s}, t-\mathrm{Bu}), 1.33(3 \mathrm{H}, \mathrm{s}$, acetonide $), 1.52(3 \mathrm{H}, \mathrm{s}$, acetonide), $3.79(1 \mathrm{H}$, br d, $J=2.5 \mathrm{~Hz}, \mathrm{H}-4), 3.92(1 \mathrm{H}$, br d, $J=12 \mathrm{~Hz}, \mathrm{H}-5), 4.05(1 \mathrm{H}, \mathrm{dd}, J=12,3 \mathrm{~Hz}, \mathrm{H}-5$ '), 5.03 $\left(1 \mathrm{H}, \mathrm{d}, J=12.5 \mathrm{~Hz},-\mathrm{CH}_{\mathrm{A}} \mathrm{H}_{\mathrm{B}} \mathrm{Ph}\right), 5.08(1 \mathrm{H}, \mathrm{d}, J=3 \mathrm{~Hz}, \mathrm{H}-2), 5.09(1 \mathrm{H}, \mathrm{d}, J=12.5 \mathrm{~Hz},-$ $\left.\mathrm{CH}_{\mathrm{A}} H_{\mathrm{B}} \mathrm{Ph}\right), 5.30\left(1 \mathrm{H}, \mathrm{d}, J=17.5 \mathrm{~Hz},-\mathrm{CH}=\mathrm{CH}_{\mathrm{A}} \mathrm{H}_{\mathrm{B}}\right), 5.35\left(1 \mathrm{H}, \mathrm{d}, J=11 \mathrm{~Hz},-\mathrm{CH}=\mathrm{CH}_{\mathrm{A}} H_{\mathrm{B}}\right), 5.88$ $(1 \mathrm{H}, \mathrm{d}, J=3 \mathrm{~Hz}, \mathrm{H}-1), 6.07\left(1 \mathrm{H}, \mathrm{dd}, J=17.5,11 \mathrm{~Hz},-\mathrm{CH}=\mathrm{CH}_{2}\right), 7.25-7.40(5 \mathrm{H}, \mathrm{m}, \mathrm{Ar}), 7.64$ $(1 \mathrm{H}$, br s, $-\mathrm{NH}) .{ }^{13} \mathrm{C} \mathrm{NMR}\left(100 \mathrm{MHz}, \mathrm{CDCl}_{3}\right) \delta-5.8,-5.6,18.1,25.6,26.2,26.7,59.3,66.3$, 68.7, 78.7, 83.4, 104.5, 112.1, 116.8, 127.0, 127.9, 128.4, 132.5, 136.7, 155.2. HRMS (FAB $\left.{ }^{+}\right)$ calcd. for $\mathrm{C}_{24} \mathrm{H}_{38} \mathrm{NO}_{6} \mathrm{Si}[\mathrm{M}+\mathrm{H}]$ 464.2468, found 464.2490.

Amine (pivalate) 17. $[\alpha]_{\mathrm{D}}^{25}+69\left(c 0.64, \mathrm{CHCl}_{3}\right)$. IR (film) $v_{\max } 3603,3381,2981,1732,1644$, $1482,1374,1285,1217,1166,1073,1017,876 \mathrm{~cm}^{-1}$. ${ }^{1} \mathrm{H}$ NMR $\left(400 \mathrm{MHz}, \mathrm{CDCl}_{3}\right) \delta 1.18(9 \mathrm{H}, \mathrm{s}$, - $\left.\mathrm{COCMe}_{3}\right), 1.32(3 \mathrm{H}, \mathrm{s}$, acetonide), $1.55(3 \mathrm{H}, \mathrm{s}$, acetonide $), 4.13(1 \mathrm{H}, \mathrm{dd}, J=11.5,6.5 \mathrm{~Hz}, \mathrm{H}-5)$, $4.18(1 \mathrm{H}, \mathrm{d}, J=3.5 \mathrm{~Hz}, \mathrm{H}-2), 4.23(1 \mathrm{H}, \mathrm{dd}, J=6.5,5 \mathrm{~Hz}, \mathrm{H}-4), 4.30(1 \mathrm{H}, \mathrm{dd}, J=11.5,5 \mathrm{~Hz}, \mathrm{H}-$ $\left.5^{\prime}\right), 5.34\left(1 \mathrm{H}, \mathrm{dd}, J=11,1 \mathrm{~Hz},-\mathrm{CH}=\mathrm{CH}_{\mathrm{A}} \mathrm{H}_{\mathrm{B}}\right), 5.45\left(1 \mathrm{H}, \mathrm{dd}, J=18,1 \mathrm{~Hz},-\mathrm{CH}=\mathrm{CH}_{\mathrm{A}} H_{\mathrm{B}}\right), 5.93$ $(1 \mathrm{H}, \mathrm{d}, J=3.5 \mathrm{~Hz}, \mathrm{H}-1), 6.09\left(1 \mathrm{H}, \mathrm{dd}, J=18,11 \mathrm{~Hz},-\mathrm{CH}=\mathrm{CH}_{2}\right) \cdot{ }^{13} \mathrm{C} \mathrm{NMR}\left(100 \mathrm{MHz}, \mathrm{CDCl}_{3}\right)$ $\delta 26.3,26.9,27.1,38.7,61.6,65.0,80.0,89.2,104.5,112.5,116.5,135.9,178.3$. HRMS (FAB $\left.{ }^{+}\right)$ calcd. for $\mathrm{C}_{15} \mathrm{H}_{26} \mathrm{NO}_{5}[\mathrm{M}+\mathrm{H}] 300.1811$, found 300.1811 .

Amine (benzoate) 18. $[\alpha]_{\mathrm{D}}^{25}+43.8\left(c 0.51, \mathrm{CHCl}_{3}\right)$. IR (film) $v_{\max } 3380,2989,1721,1603$, $1453,1374,1317,1275,1216,1166,1100,1071,1018,931,876,805 \mathrm{~cm}^{-1}$. ${ }^{1} \mathrm{H}$ NMR $(300 \mathrm{MHz}$, $\left.\mathrm{CDCl}_{3}\right) \delta 1.33(3 \mathrm{H}, \mathrm{s}$, acetonide), $1.58(3 \mathrm{H}, \mathrm{s}$, acetonide $), 4.20(1 \mathrm{H}, \mathrm{d}, J=3.5 \mathrm{~Hz}, \mathrm{H}-2), 4.36-$ $4.44(2 \mathrm{H}, \mathrm{m}, \mathrm{H}-4, \mathrm{H}-5), 4.54(1 \mathrm{H}, \mathrm{dd}, J=14,7 \mathrm{~Hz}, \mathrm{H}-5$ '), $5.32(1 \mathrm{H}, \mathrm{dd}, J=11,1 \mathrm{~Hz},-$ $\left.\mathrm{CH}=\mathrm{CH}_{\mathrm{A}} \mathrm{H}_{\mathrm{B}}\right), 5.47\left(1 \mathrm{H}, \mathrm{dd}, J=17.5,1 \mathrm{~Hz},-\mathrm{CH}=\mathrm{CH}_{\mathrm{A}} H_{\mathrm{B}}\right), 5.99(1 \mathrm{H}, \mathrm{d}, J=4 \mathrm{~Hz}, \mathrm{H}-1), 6.14(1 \mathrm{H}$, $\left.\mathrm{dd}, J=17.5,11 \mathrm{~Hz},-\mathrm{CH}=\mathrm{CH}_{2}\right), 7.40-7.46(2 \mathrm{H}, \mathrm{m}, \mathrm{Ar}), 7.56(1 \mathrm{H}, \mathrm{tt}, J=7,1.5 \mathrm{~Hz}, \mathrm{Ar}), 8.00-8.06$ $(2 \mathrm{H}, \mathrm{m}, \mathrm{Ar}) .{ }^{13} \mathrm{C} \mathrm{NMR}\left(75 \mathrm{MHz}, \mathrm{CDCl}_{3}\right) \delta 26.2,26.8,62.2,65.1,80.0,89.2,104.7,112.6$, $116.5,128.4,129.8,133.1,135.9,166.5$. HRMS $\left(\mathrm{FAB}^{+}\right)$calcd. for $\mathrm{C}_{17} \mathrm{H}_{22} \mathrm{NO}_{5}[\mathrm{M}+\mathrm{H}] 320.1498$, found 320.1491 .

Urea (benzoate) 19. $[\alpha]_{\mathrm{D}}{ }^{26}+26.1\left(c 0.75, \mathrm{CHCl}_{3}\right) . \quad$ IR (film) $v_{\max } 3379,2990,1722,1603,1535$, 1454, 1375, 1274, 1166, 1073, 1004, 928, $877 \mathrm{~cm}^{-1}$. ${ }^{1} \mathrm{H}$ NMR $\left(400 \mathrm{MHz}, \mathrm{CDCl}_{3}\right) \delta 1.34(6 \mathrm{H}, \mathrm{s}$, acetonide), $1.56(6 \mathrm{H}, \mathrm{s}$, acetonide), 4.32-4.41 (4H, m, H-4 \& H5), $4.64(2 \mathrm{H}, \mathrm{dd}, J=8,6.5 \mathrm{~Hz}, \mathrm{H}-$ 5'), $5.10(2 \mathrm{H}, \mathrm{d}, J=3.5 \mathrm{~Hz}, \mathrm{H}-2), 5.33-5.41(2 \mathrm{H}, \mathrm{br},-\mathrm{NH}), 5.35(2 \mathrm{H}, \mathrm{d}, J=17.5 \mathrm{~Hz},-$ $\left.\mathrm{CH}=\mathrm{CH}_{\mathrm{A}} \mathrm{H}_{\mathrm{B}}\right), 5.37\left(2 \mathrm{H}, \mathrm{d}, J=11 \mathrm{~Hz},-\mathrm{CH}=\mathrm{CH}_{\mathrm{A}} H_{\mathrm{B}}\right), 5.90(2 \mathrm{H}, \mathrm{d}, J=3.5 \mathrm{~Hz}, \mathrm{H}-1), 6.14(2 \mathrm{H}, \mathrm{dd}$, $\left.J=17.5,11 \mathrm{~Hz},-\mathrm{CH}=\mathrm{CH}_{2}\right), 7.44(4 \mathrm{H}$, br t $, J=7.5,-\mathrm{Ar}), 7.56(2 \mathrm{H}, \mathrm{tt}, J=7.5,1.5 \mathrm{~Hz},-\mathrm{Ar}), 8.00$ $(4 \mathrm{H}, \mathrm{m},-\mathrm{Ar}) .{ }^{13} \mathrm{C} \mathrm{NMR}\left(100 \mathrm{MHz}, \mathrm{CDCl}_{3}\right) \delta 26.3,26.8,61.0,68.0,78.9,84.1,104.8,112.6$, 117.4, 128.6, 129.4, 129.7, 132.5, 133.5, 155.8, 166.3. HRMS $\left(F A B^{+}\right)$calcd. for $\mathrm{C}_{35} \mathrm{H}_{41} \mathrm{~N}_{2} \mathrm{O}_{11}$ $[\mathrm{M}+\mathrm{H}]$ 665.2711, found 665.2728.

Cyclic carbamate 21. $[\alpha]_{\mathrm{D}}^{23}+88\left(c 0.25, \mathrm{CHCl}_{3}\right)$. IR (film) $v_{\max } 3229,3020,1707,1560,1508$, 1465, 1425, 1322, 1216, 1080, $999 \mathrm{~cm}^{-1} .{ }^{1} \mathrm{H} \mathrm{NMR}$ (400 MHz, $\left.\mathrm{CDCl}_{3}\right) \delta 1.35$ (3H, s, acetonide), $1.57(3 \mathrm{H}, \mathrm{s}$, acetonide $), 4.16(1 \mathrm{H}, \mathrm{dd}, J=12.5,1 \mathrm{~Hz}, \mathrm{H}-5), 4.22(1 \mathrm{H}$, br s, H-4), $4.38(1 \mathrm{H}, \mathrm{d}, J=$ $3 \mathrm{~Hz}, \mathrm{H}-2), 4.43\left(1 \mathrm{H}, \mathrm{dd}, J=12.5,1.5 \mathrm{~Hz}, \mathrm{H}-5\right.$ '), $5.49\left(1 \mathrm{H}, \mathrm{d}, J=10.5 \mathrm{~Hz}, \mathrm{CH}=\mathrm{CH}_{\mathrm{A}} \mathrm{CH}_{\mathrm{B}}\right), 5.52$ $\left(1 \mathrm{H}, \mathrm{d}, J=17 \mathrm{~Hz}, \mathrm{CH}=\mathrm{CH}_{\mathrm{A}} \mathrm{CH}_{\mathrm{B}}\right), 5.97(1 \mathrm{H}, \mathrm{d}, J=3 \mathrm{~Hz}, \mathrm{H}-1), 6.13(1 \mathrm{H}, \mathrm{dd}, J=17,10.5 \mathrm{~Hz}$, 
$\left.\mathrm{CH}=\mathrm{CH}_{\mathrm{A}} \mathrm{CH}_{\mathrm{B}}\right) 6.90(1 \mathrm{H}$, br s, $-\mathrm{NH}) .{ }^{13} \mathrm{C} \mathrm{NMR}\left(75 \mathrm{MHz}, \mathrm{CDCl}_{3}\right) \delta$ 26.4, 27.0, 63.6, 66.9, 73.2, 86.8, 105.2, 113.3, 119.6, 133.5, 153.3. HRMS $\left(\mathrm{FAB}^{+}\right)$calcd. for $\mathrm{C}_{11} \mathrm{H}_{16} \mathrm{NO}_{5}[\mathrm{M}+\mathrm{H}]$ 242.1028, found 242.1023. 